\title{
Mathematical model for the population dynamics of the Serengeti ecosystem
}

\author{
Janeth James Ngana ${ }^{1}$, Livingstone Serwadda Luboobi ${ }^{2}$, Dmitry Kuznetsov ${ }^{1}$ \\ ${ }^{1}$ Nelson Mandela African Institution of Science and Technology (NM-AIST), Arusha, Tanzania \\ ${ }^{2}$ Department of Mathematics, Makerere University, Kampala, Uganda

\section{Email address:} \\ nganaj@nm-aist.ac.tz (J. J. Ngana), luboobi@cns.mak.ac.ug (L. S. Luboobi), dmitry.kuznetsov@nm-aist.ac.tz (D. Kuznetsov)
}

\section{To cite this article:}

Janeth James Ngana, Livingstone Serwadda Luboobi, Dmitry Kuznetsov. Mathematical Model for the Population Dynamics of the Serengeti Ecosystem. Applied and Computational Mathematics. Vol. 3, No. 4, 2014, pp. 171-176.

doi: $10.11648 /$ j.acm.20140304.18

\begin{abstract}
Several ecological studies have tried to model the population dynamics of the ungulate migratory animals individually without including the food and predation factors in the models. In this paper, we analyze the population dynamics for herbivores, carnivores and the grass volume using the secondary data from the years 1996-2006. The lions' data didn't correlate with the model. Due to that, the sensitivity analysis was carried out for the parameters. The herbivores predation on grass reduces the volume of grass. The crocodile predation on herbivores decreases the population of herbivores. Then the crocodile population increases, when its' natural death rate in the absence of prey decreases. The herbivores population increases as its' intrinsic logistic rate increases. There is a trend of Grass periodic increase and decrease as the rainfall constant value changes periodically. The herbivores population decreases as the lion predation on them increases. And lastly, the lions' population decreases as the natural death rate of lion in the absence of prey increased.
\end{abstract}

Keywords: Carnivores, Herbivores, Grass, Population Dynamics, Migration, Ecosystem, Wildebeest

\section{Introduction}

There is nowhere else in the world where there is such a movement of animals as immense as the wildebeests (Connochaetes taurinus), zebras (Equus burchelli) and Thomson's gazelle (Gazella thomsoni), migrating from Serengeti National Park in Tanzania to Masai Mara National Reserve in Kenya and back. The wildebeest (Connochaetes taurinus) migration in the Serengeti/ Mara ecosystem of Tanzania and Kenya represents an iconic example of ungulate migration and constitutes one of the most thoroughly documented animal migrations in one of the most intensively studied ecosystems on earth $[13,8,2,9$, $16,17,15,10]$. The migration is driven by a marked, highly seasonal rainfall gradient, increasing from South East to North West, coupled with strong differences in soil fertility and plant nutritional content between the grassland and savanna habitats $[8,6,5,11]$. Speaking of population trends and predation, by using a logistic model equation for estimating the wildebeest's population, from 1960s to 1980s, the Serengeti-Mara ecosystem witnessed dramatic changes. By mid-1970s, the wildebeest population increased by a factor of five, and remained at approximately 1.3 million with slight variations $[14,3,12]$. Reconstruction of 100 years of the vegetation dynamics in the Serengeti ecosystem gives an insight of what might happen if the wildebeest population is reduced to about 200,000 , as it is believed to have been following the Rinderpest epidemic in the early 1900s [4].

The Thomson's gazelles population has been declining almost two thirds over a decade ago. This decline has been due to: predation, interspecific competition and diseases. Predation has been found to be the main factor preventing the increase of the Thomson's gazelles population at the Serengeti National Park [1].

Imposing seasonal variation to multispecies models that in a constant environment tend towards a stable equilibrium can lead to cycles and even chaotic dynamics [7], with overcompensating density dependence leading to low population densities where extinction may be risked.

In this paper we formulate and analyze a population dynamics model that depicts the food chain relationship between: grass; herbivores (wildebeest, zebra and Thomson's gazelles); carnivore predators (lions and crocodiles); thus reflecting on grass food factor, the 
herbivores population size as well as the impact of predation at the Serengeti ecosystem.

\section{Model Formulation}

In the formulation of the model we make the following assumptions:

\subsection{Assumptions}

1) All the herbivores considered were adults non-residents.

2) All the herbivores factually considered were from Tanzania.

3) All the herbivores considered were diseased free.

4) There was no climate change during the research time.

5) There was no drought during the research time.

6) There was no poaching.

7) The predators considered were lions and croco diles.

\subsection{The Variables}

The following variables represent the sub-populations of the ecosystem as described in section 2.1:

$$
\begin{aligned}
& G_{T}=\text { grass vegetation } \\
& W_{T}=\text { wildebeest } \\
& Z_{T}=\text { zebra } \\
& A_{T}=\text { Thomson's gazelles } \\
& L_{T}=\text { lions } \\
& C=\text { crocodiles }
\end{aligned}
$$

\subsection{The Model}

Applying the assumptions in Section 2.1 and the variables defined in Section 2.2 we derive the model for the ecosystem to consist of the following six ordinary differential equations: (1) - (6):

$$
\begin{gathered}
\frac{d G_{T}}{d t}=k_{0} \cos (\omega t) G_{T}-\alpha_{T 0} W_{T} G_{T}-\alpha_{T 1} Z_{T} G_{T}-\propto_{T 2} A_{T} G_{T} \\
\frac{d W_{T}}{d t}=\eta_{0} G_{T} W_{T}+\lambda_{T 0} W_{T}-\mu_{T 0} W_{T}-\omega_{T 0} L_{T} W_{T}-\beta_{T 0} C W_{T} \\
\frac{d Z_{T}}{d t}=\eta_{1} G_{T} Z_{T}+\lambda_{T 1} Z_{T}-\mu_{T 1} Z_{T}-\omega_{T 1} L_{T} Z_{T}-\beta_{T 1} C Z_{T} \\
\frac{d A_{T}}{d t}=\eta_{2} G_{T} A_{T}+\lambda_{T 2} Z_{T}-\mu_{T 2} A_{T}-\omega_{T 2} L_{T} A_{T}-\beta_{T 2} C A_{T} \\
\frac{d L_{T}}{d t}=-\phi L_{T}+v_{0} W_{T} L_{T}+v_{1} Z_{T} L_{T}+v_{2} A_{T} L_{T} \\
\frac{d C}{d t}=-\varepsilon C+\delta_{0} W_{T} C+\delta_{1} Z_{T} C+\delta_{2} A_{T} C
\end{gathered}
$$

In which $\mathrm{T}$ stands for Tanzania and the parameters are defined as follows:

\begin{tabular}{cl}
\hline Parameter & Abbreviation \\
\hline$k_{0}$ & the rainfall constant ratio \\
$\omega$ & angle in radians \\
$\alpha_{T 0}$ & the efficiency rate of wildebeest predation on \\
$\alpha_{T 1}$ & the efficiency rate of zebra predation on grass
\end{tabular}

the efficiency rate of Thomson's gazelles predation on grass

the efficiency rate of grass prey by wildebeest

$\eta_{0}$

$\lambda_{T 0}$

$\mu_{T 0}$

$\omega_{T 0}$

$\beta_{T 0}$

$\eta_{1}$

$\lambda_{T 1}$

$\mu_{T 1}$

$\omega_{T 1}$

$\beta_{T 1}$

$\eta_{2}$

$\lambda_{T 2}$

$\mu_{T 2}$

$\omega_{T 2}$

$\beta_{T 2}$

$\phi$

$v_{0}$

$v_{0}$

$v_{1}$

$v_{2}$

$\varepsilon$

$\delta_{0}$

$\delta_{1}$

$\delta_{2}$ the natural birth rate of the wildebeest

the natural mortality rate of the wildebeest

the efficiency rate of lion predation on the wildebeest

the efficiency rate of crocodile predation on the wildebeest

the efficiency rate of grass prey by zebra

the natural birth rate of the zebra

the natural mortality rate of the zebra

the efficiency rate of lion predation on the zebra

the efficiency rate of crocodile predation on the zebra

the efficiency rate of grass prey by Thomson's gazelles

the natural birth rate of the Thomson's gazelles

the natural mortality rate of the Thomson's Gazelles

the efficiency rate of lion predation on the Thomson's gazelles

the efficiency of crocodile predation on the Thomson's gazelles

natural death rate of lions in the absence of prey

the efficiency rate of the lion in the presence of wildebeest

the efficiency rate of the lion in the presence of zebra

the efficiency and rate of the lion in the presence of Thomson's gazelle

natural death rate of crocodiles in the absence of prey

the efficiency rate of the crocodiles in the presence of wildebeest

the efficiency rate of the crocodiles in the presence of zebra

the efficiency rate of the crocodiles in the presence of Thomson's gazelle

\subsection{Model Analysis}

To analyze the model, MAPLE software was used. MAPLE was used to find the equilibrium points, Jacobian matrices, the eigenvalues as well as the stability of the equilibrium points.

\subsubsection{Equilibrium Points}

Some of the equilibrium points were:

$$
\left\{C=0, A_{T}=0, G_{T}=0, L_{T}=0, W_{T}=0, Z_{T}=0\right\},
$$




$$
\begin{aligned}
& \left\{C=0, A_{T}=\frac{k_{0} \cos (\omega t)}{\alpha_{T 2}}, G_{T}=-\frac{\lambda_{T 2}-\mu_{T 2}}{\eta_{2}}, L_{T}=0, W_{T}=0, Z_{T}\right. \\
& =0\} \\
& \left\{C=\frac{\lambda_{T 2}-\mu_{T 2}}{\beta_{T 2}}, A_{T}=\frac{\varepsilon}{\delta_{2}}, G_{T}=0, L_{T}=0, W_{T}=0, Z_{T}=0\right\} \\
& \left\{C=\frac{\lambda_{T 1}-\mu_{T 1}}{\beta_{T 1}}, A_{T}=0, G_{T}=0, L_{T}=0, W_{T}=0, Z_{T}=\frac{\varepsilon}{\delta_{1}}\right\} \text {, } \\
& \left\{C=\frac{\lambda_{T 0}-\mu_{T 0}}{\beta_{T 0}}, A_{T}=0, G_{T}=0, L_{T}=0, W_{T}=\frac{\varepsilon}{\delta_{0}}, Z_{T}=0\right\}, \\
& \left\{C=0, A_{T}=0, G_{T}=0, L_{T}=\frac{\lambda_{T 1}-\mu_{T I}}{\omega_{T I}}, W_{T}=0, Z_{T}=\frac{\phi}{v_{1}}\right\}
\end{aligned}
$$

\subsubsection{The Jacobian Matrices}

Jacobian matrices were then found using the same MAPLE software.

Some of those matrices were:

$$
J 1:=\left[\begin{array}{cccccc}
k_{0} \cos (\omega t) & 0 & 0 & 0 & 0 & 0 \\
0 & \lambda_{T 0}-\mu_{T 0} & 0 & 0 & 0 & 0 \\
0 & 0 & \lambda_{T 1}-\mu_{T 1} & 0 & 0 & 0 \\
0 & 0 & 0 & \lambda_{T 2}-\mu_{T 2} & 0 & 0 \\
0 & 0 & 0 & 0 & -\phi & 0 \\
0 & 0 & 0 & 0 & 0 & -\varepsilon
\end{array}\right]
$$$$
J 2:=\left[\left[0, \frac{\alpha_{T 0}\left(\lambda_{T 2}-\mu_{T 2}\right)}{\eta_{2}}, \frac{\alpha_{T 1}\left(\lambda_{T 2}-\mu_{T 2}\right)}{\eta_{2}}, \frac{\alpha_{T 2}\left(\lambda_{T 2}-\mu_{T 2}\right)}{\eta_{2}},\right.\right.
$$

$$
0,0] \text {, }
$$$$
\begin{aligned}
& {\left[0,-\frac{\eta_{0}\left(\lambda_{T 2}-\mu_{T 2}\right)}{\eta_{2}}+\lambda_{T 0}-\mu_{T 0}, 0,0,0,0\right],} \\
& {\left[0,0,-\frac{\eta_{1}\left(\lambda_{T 2}-\mu_{T 2}\right)}{\eta_{2}}+\lambda_{T 1}-\mu_{T 1}, 0,0,0\right],}
\end{aligned}
$$$$
\left[\frac{\eta_{2} k_{0} \cos (\omega t)}{\alpha_{T 2}}, 0,0,0,-\frac{\omega_{T 2} k_{0} \cos (\omega t)}{\alpha_{T 2}},-\frac{\beta_{T 2} k_{0} \cos (\omega t)}{\alpha_{T 2}}\right.
$$$$
\begin{aligned}
& {\left[0,0,0,0,-\phi+\frac{v_{2} k_{0} \cos (\omega t)}{\alpha_{T 2}}, 0\right]} \\
& \left.\left[0,0,0,0,0,-\varepsilon+\frac{\delta_{2} k_{0} \cos (\omega t)}{\alpha_{T 2}}\right]\right]
\end{aligned}
$$

$$
\begin{aligned}
& J 3:=\left[\left[0, \frac{\alpha_{T 0}\left(\lambda_{T 1}-\mu_{T 1}\right)}{\eta_{1}}, \frac{\alpha_{T 1}\left(\lambda_{T 1}-\mu_{T 1}\right)}{\eta_{1}}, \frac{\alpha_{T 2}\left(\lambda_{T 1}-\mu_{T 1}\right)}{\eta_{1}},\right.\right. \\
& 0,0] \text {, } \\
& \begin{array}{l}
{\left[0,-\frac{\eta_{0}\left(\lambda_{T 1}-\mu_{T 1}\right)}{\eta_{1}}+\lambda_{T 0}-\mu_{T 0}, 0,0,0,0\right],} \\
{\left[\frac{\eta_{1} k_{0} \cos (\omega t)}{\alpha_{T 1}}, 0,0,0,-\frac{\omega_{T 1} k_{0} \cos (\omega t)}{\alpha_{T 1}},-\frac{\beta_{T 1} k_{0} \cos (\omega t)}{\alpha_{T 1}}\right.}
\end{array} \\
& {\left[0,0,0,-\frac{\eta_{2}\left(\lambda_{T 1}-\mu_{T 1}\right)}{\eta_{1}}+\lambda_{T 2}-\mu_{T 2}, 0,0\right] \text {, }} \\
& \begin{array}{l}
{\left[0,0,0,0,-\phi+\frac{v_{1} k_{0} \cos (\omega t)}{\alpha_{T 1}}, 0\right]} \\
\left.\left[0,0,0,0,0,-\varepsilon+\frac{\delta_{1} k_{0} \cos (\omega t)}{\alpha_{T 1}}\right]\right]
\end{array}
\end{aligned}
$$

\subsubsection{The Eigenvalues}

Using MAPLE we get twenty eigenvalues for the equilibrium solution (of which only one equilibrium point was stable).

The eigenvalue for the first equilibrium point is:

$$
\left[\begin{array}{c}
-\varepsilon \\
k_{0} \cos (\omega t) \\
\lambda_{T 0}-\mu_{T 0} \\
\lambda_{T 1}-\mu_{T 1} \\
\lambda_{T 2}-\mu_{T 2} \\
-\phi
\end{array} \mid\right.
$$

$\therefore$ This is a stable equilibrium point iff: $\varepsilon>0$ and $\phi>0$,

$$
\cos (\omega t)<0,
$$

Meaning: in the absence of prey, crocodiles should naturally die, lions should also die naturally and rainfall should vary periodically respectively.

$$
\left(\lambda_{T 0}-\mu_{T 0}\right)<0,
$$

Meaning: the Wildebeest birth rate should be less than its mortality rate.

$$
\left(\lambda_{T 1}-\mu_{T 1}\right)<0,
$$

Meaning: the Zebra birth rate should be less than its mortality rate.

$$
\left(\lambda_{T 2}-\mu_{T 2}\right)<0 .
$$

Meaning: the Thomson's Gazelles' birth rate should be less than its mortality rate.

All these conditions are not likely to occur simultaneously. Hence we may conclude that it is very unlikely for the Serengeti ecosystem will go to extinction at any time unless disaster enforces the conditions occur. 


\section{Simplification of the Model}

We had to simplify the model due to the MATLAB software failure in finding the exceeding large number of parameters.

This was done using the following steps:

From the system of equations (1) - (6), for example the birth and mortality rates are combined together to form the intrinsic rates of growth:

Thus, dropping the subscript " $T$ ", we have equations

Refer to equations (2) - (4);

Let the intrinsic rates of growth be:

$$
\begin{aligned}
& \left(\lambda_{0}-\mu_{0}\right)=\gamma_{0} \\
& \left(\lambda_{1}-\mu_{1}\right)=\gamma_{1} \\
& \left(\lambda_{2}-\mu_{2}\right)=\gamma_{2}
\end{aligned}
$$

for the herbivores equations respectively.

Let the effect of grass prey on Wildebeest, Zebra and Gazelle Thomson's respectively be the same as $\eta$, thus:

$$
\begin{gathered}
\eta_{0}=\eta_{1}=\eta_{2}=\eta \\
\omega_{0}=\omega_{1}=\omega_{2}=\omega ; \quad \beta_{0}=\beta_{1}=\beta_{2}=\beta \\
\gamma_{0}=\gamma_{1}=\gamma_{2}=\gamma .
\end{gathered}
$$

Let $H=W+Z+A$

$$
\text { ( } H=\text { Herbivores })
$$

Hence: $\frac{d H}{d t}=\frac{d W}{d t}+\frac{d Z}{d t}+\frac{d A}{d t}$

Let $\alpha_{0}=\alpha_{1}=\alpha_{2}=\alpha$;

$$
\mathrm{v}_{0}=\mathrm{v}_{1}=\mathrm{v}_{0}=v ; \quad \delta_{0}=\delta_{1}=\delta_{2}=\delta
$$

After the simplification of the model, the simplified system becomes:

$$
\begin{aligned}
\frac{d G}{d t} & =k_{0} \cos (\theta t) G-\alpha H G \\
\frac{d H}{d t} & =\eta G H+\gamma H-\omega L H-\beta C H \\
\frac{d L}{d t} & =-\phi L+v H L \\
\frac{d C}{d t} & =-\varepsilon C+\delta H C
\end{aligned}
$$

The variables are:

$G=$ grass

$H=$ herbivores

$L=$ lions

$C=$ crocodiles

The parameters are now eleven, where:

\section{Parameter}

$k_{0}$

Abbreviation

$k_{0}$ the rainfall constant

$\alpha$

$\begin{array}{ll}\theta & \begin{array}{l}\text { the angle in radians } \\ \text { the interaction rate of grass prey on } \\ \gamma\end{array} \\ \omega & \begin{array}{l}\text { herbivores } \\ \text { the intrinsic logistic rate } \\ \text { the efficiency rate of lion predation on } \\ \text { herbivores } \\ \text { the efficiency rate of crocodile predation } \\ \text { on herbivores } \\ \text { the natural death rate of lions in the } \\ \text { absence of prey } \\ \text { the efficiency rate of lion in the presence } \\ \text { of herbivores } \\ \text { the natural death rate of crocodile in the } \\ \text { absence of prey } \\ \text { the efficiency rate of the crocodile in the } \\ \text { presence of herbivores }\end{array} \\ \end{array}$

Only three graphs (for Grass, Herbivores and Crocodiles) fit the model, while the one Lions does not, this means the data does not correlate with the model. Sensitivity Analysis is then applied.

\section{Sensitivity Analysis}

For this analysis the eight estimated parameters were altered and observed.

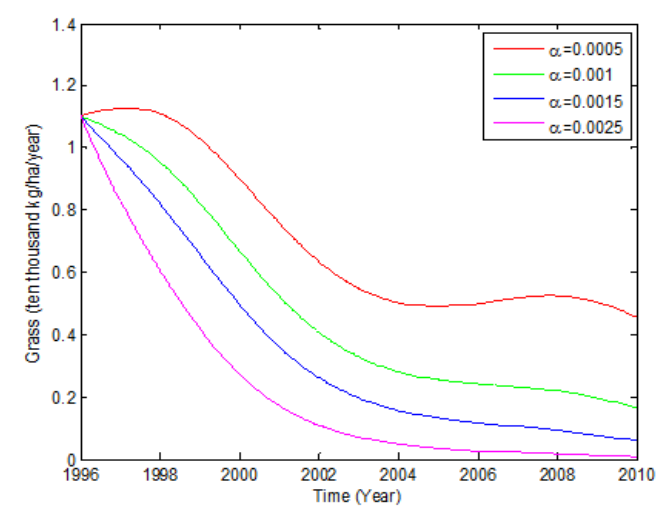

Figure 4.1(a). As the effect of herbivores predation on grass increased, the volume of grass decreased from 1996 to 2010.

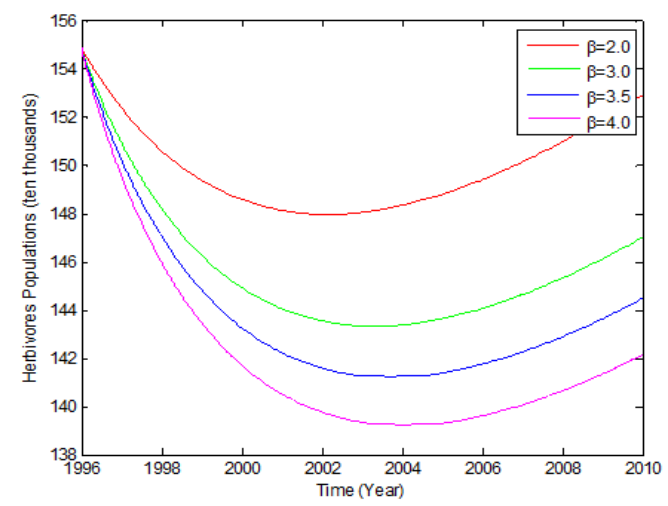

Figure 4.1(b). As the effect of crocodile predation on herbivores increased, the population of herbivores decreased from 1996 to 2004. But the population increased afterwards from 2004 to 2010 as the predation rate decreased. 


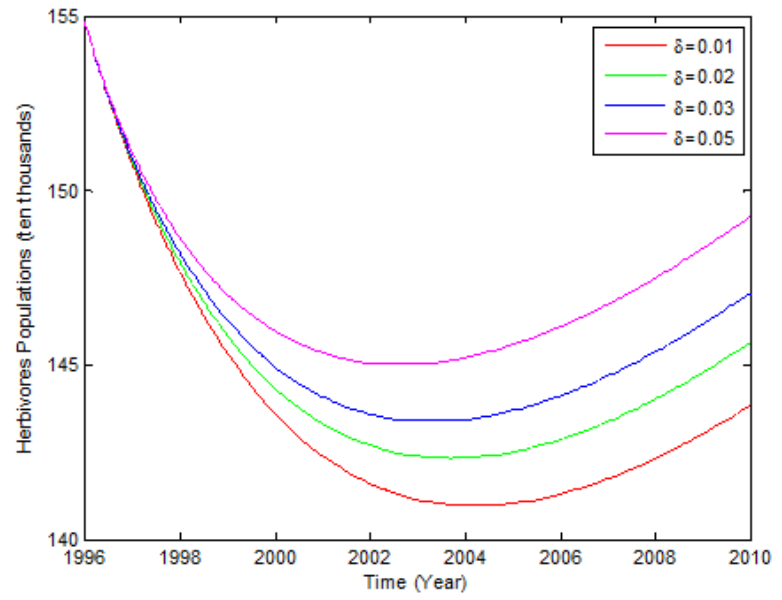

Figure 4.1(c). As the effect of crocodile predation on herbivores increased, the herbivores population decreased from 1996 to 2004, but then the population increased from 2004 to 2010 as the rate decreased.

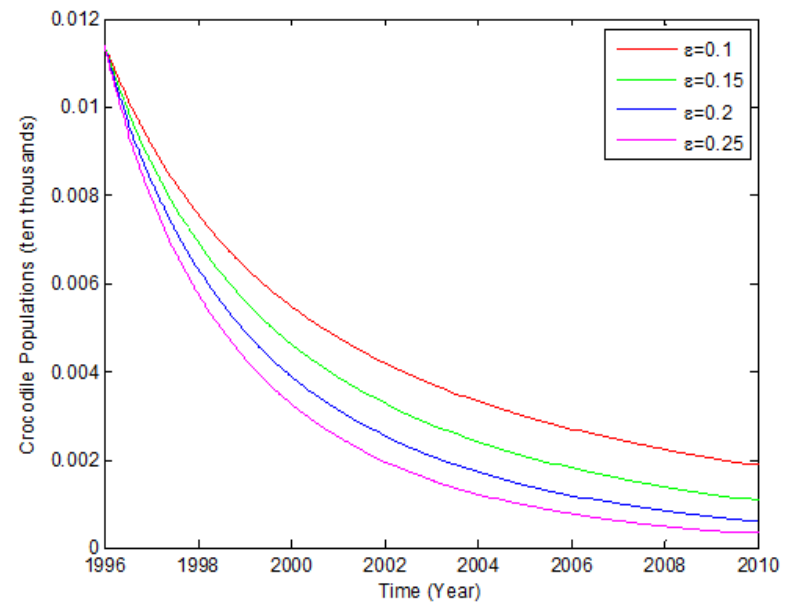

Figure 4.1(d). As the natural death rate of crocodile in the absence of prey increased from 1996 to 2010, then the crocodile population decreased.

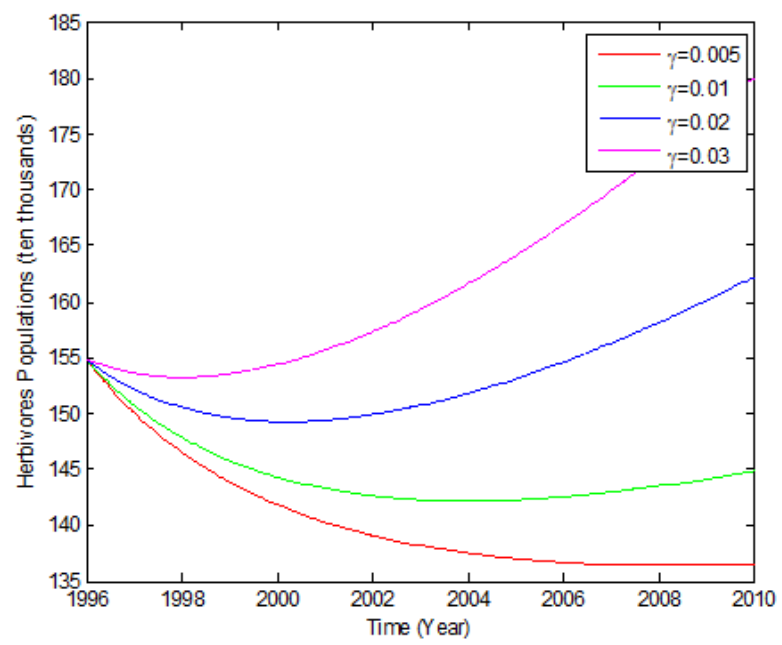

Figure 4.1(e). As the intrinsic logistic rate of growth decreased slightly variably from 1996 to 2008 at maximum, the herbivores population decreased, but the population increased variably from 1998 onwards as the rate increased.

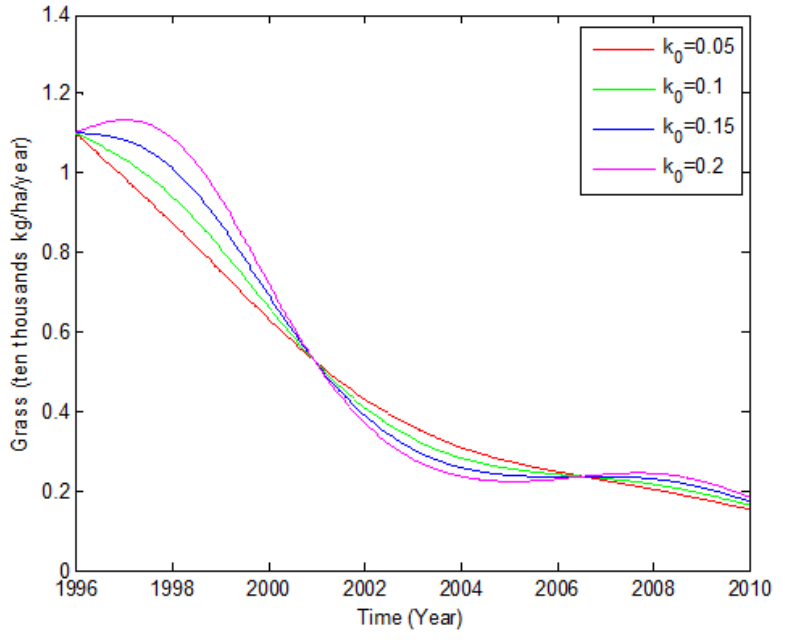

Figure 4.1(f). There is a trend of grass periodic increase and decrease as the $k$ value increased and decreased periodically. Thus, from 1996 to 2001 the grass volume increased due to the increased in rainfall. But from 2001 to 2006 the volume grew less again.

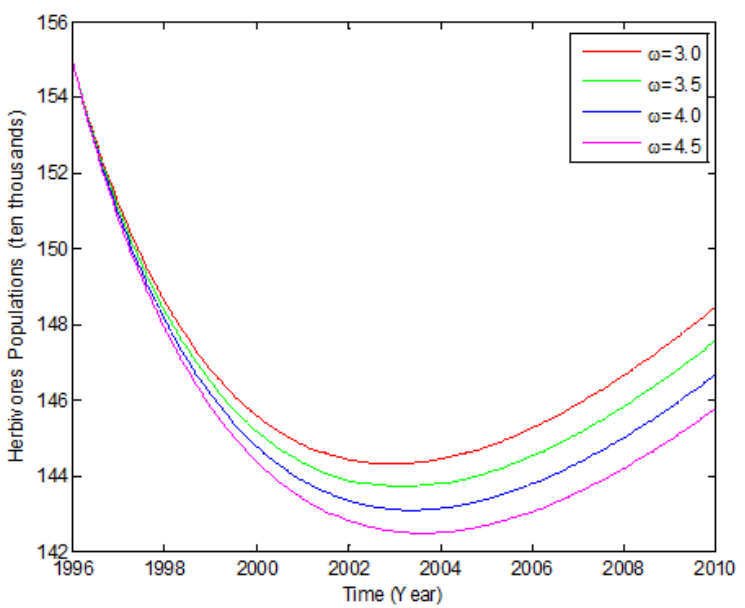

Figure 4.1(g). As the effect of lion predation on herbivores increased from 1996 to 2003, the herbivores population decreased. But the population increased again from 2003 onwards as the rate of lion predation started to decrease again.

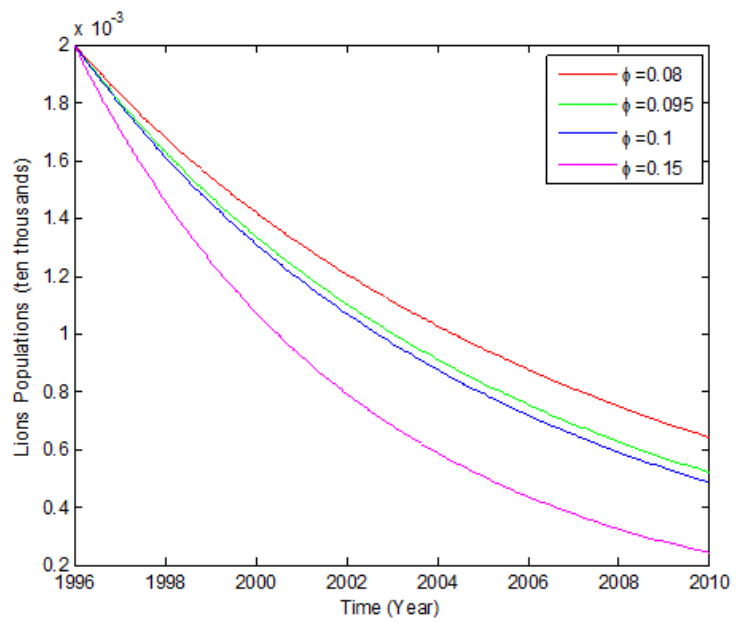

Figure 4.1(h). As the natural death rate of lion in the absence of prey increased from 1996 to 2010, the lions' population happened to decrease onwards. 


\section{Conclusion}

In this study, the sensitivity analysis was carried out from 1996 to 2010 because the lion's population didn't fit the model due to scarcity of data. Eight sensitive parameters to the model were analyzed. The interaction between the herbivores and grass as food, resulted into the decrease of the volume of the grass. The predation on herbivores by crocodiles decreased the population of the herbivores. The crocodile population increased as its natural death rate decreased. The herbivores population increased as its intrinsic logistic rate increased. The grass grew periodically due to rainfall seasons. The existence of lions depended much on their predation on herbivores.

\section{References}

[1] Borner, M., FitzGibbon, C. D., Borner, Mo., Caro, T. M., Lindsay, W. K., Collins, D. A., Holt, M. E., (1987). "The decline of the Serengeti Thomson's gazelle population." Oecologia 73 (1): 32-40.

[2] Fryxell, J. M., Greever, J., and Sinclair, A. R. E. (1988). Causes and consequences of migration in large herbivores. Trends in Ecology and Evolution 3: 237-241.

[3] Dublin, H.T., Sinclair, A.R.E., Boutin, S., Anderson, E., Jago, M. \& Arcese, P. (1990) Does competition regulate ungulate populations? Further evidence from Serengeti, Tanzania. Oecologia, 82, 283-288.

[4] Dublin, H. T. (1995). Vegetation dynamics in the SerengetiMara ecosystem: The role of elephants, fi re, and other factors. In Serengeti II, ed. A. R. E. Sinclair and P. Arcese, 71-90. Chicago: University of Chicago Press.

[5] Holdo, R. M., Holt, R. D., Sinclair, A. R., Godley, B. J., \& Thirgood, S. (2011). Migration impacts on communities and ecosystems: empirical evidence and theoretical insights. Animal Migration: A Synthesis, 131-143.

[6] Holdo, R. M., Holt, R. D., and Fryxell, J.M. (2009). Opposing rainfall and pant nutritional gradients best explain the wildebeest migration in the Serengeti. The American
Naturalist, 173 (4), 431-445.

[7] King, A. A. and Schaffer. W. M. (1999). The rainbow bridge: Hamiltonian limits and reso-nance in predator-prey dynamics. J. Math. Biol. 39: 439-469.

[8] Maddock, L., Sinclair, A. R. E and Norton-Griffiths, M. (1979). The Migration and Grazzing succession in Serengeti: Dynamics of an Ecosystem. 104-29. Chicago: University of Chicago Press.

[9] Mduma S.A.R., Sinclair A.R.E. AND Hiborn, R. (1999). Food regulates the Serengeti Wildbeest: a 40-year record. Journal of Animal Ecology, 68, 1101-1122

[10] Musiega, D. E., and Kazadi, S.N. (2004). Simulating the East African Wildebeest Migration Patterns using GIS and remote sensing. African Journal of Ecology, 42 (4), 355-362.

[11] Ngana, J. J., Luboobi, L.S, Kuznetsov, D. (2014). Modelling the Migratory Population Dynamics of the Serengeti Ecosystem. Applied and Computational Mathematics. Vol. 3, No. 4, 2014, pp. 125-129.

[12] Onyeanusi, A.E. (1989). Large herbivore grass take-off in Masai-Mara National Reserve:Implications for the Serengeti-Mara migrants. J. Arid Environ. 16, 203-209.

[13] Pennycuick, C.J. (1975). On the running of gnu (Connochaetes taurinus) and other animals. Journal of Experimental Biology. 63, p.775-799.

[14] Sinclair, A. (2003). Patterns of predation in a diverse predator- prey system. Nature, 425, 288-290.

[15] Sinclair, A.R.E. and Norton-Griffiths, M. (1979). Serengeti: Dynamics of an Ecosystem. Univ. Chicago Press, Chicago, USA.

[16] Wilmhurst, J. F. Fryxell, J. M., Fram, B.P., Sinclair, A. R. E., Henschel, C. P. (1999). Spatial Distribution of Serengeti Wildebeest in relation to Resources. Can. J. Zool.77, $1223-1232$.

[17] Wolanski, E.J and Gereta, E (2001) Water quantity and quality as the factors driving the Serengeti ecosystem, Tanzania. Hydrobiologia. 458: 169-180. 